\title{
Recombination Line Abundances in NGC 6210
}

\author{
R. L. Kingsburgh ${ }^{1}$, M. Komljenovic ${ }^{1}$, J. A. López ${ }^{2}$ and M. Peimbert ${ }^{2}$ \\ ${ }^{1}$ York University, Dept. of Physics and Astronomy; \\ ${ }^{2}$ Universidad Nacional Autónoma de México, Instituto de Astronomia
}

We present abundances derived from echelle spectroscopy of the planetary nebula NGC 6210 from the forbidden, collisionally excited lines, and from the permitted, recombination lines. We have obtained spectra from 3 positions in the nebula: at the central star, and 6 and 12 arcseconds south of the central star. We find a discrepancy of a factor of 2 between the $\mathrm{O}^{2+} / \mathrm{H}^{+}$ratio derived by the recombination lines and by the forbidden lines in each spatial position. This discrepancy may result from the presence of temperature fluctuations in NGC 6210, where the collisionally excited lines are exponentially weighted to regions of higher temperature. A value for the root-mean-square temperature fluctuation parameter $t^{2}\left(\begin{array}{l}O I I \\ a b u n\end{array}\right)=0.038_{-0.009}^{+0.007}$ is derived for the central star spectrum, $0.040_{-0.013}^{+0.006}$ for the 6 arcsecond offset, and $0.051_{-0.015}^{+0.014}$ for the 12 arcsecond offset spectrum. Thus no significant variation in $t^{2}$ is seen as a function of spatial position within the nebula. We have also estimated $t^{2}$ by comparing the derived Balmer Jump temperature at the 6 arcsecond offset, $\mathrm{T}_{\mathrm{e}}(\mathrm{BaJ})=7900_{-1600}^{+2400} \mathrm{~K}$, with the temperature derived from the [O III] 5007/4363 $\mathrm{\text {ratio }}$ $\mathrm{T}_{\mathrm{e}}[\mathrm{O} \mathrm{III}]=9370 \pm 100 \mathrm{~K}$ at this position. This comparison suggest $t^{2}(\mathrm{BaJ})=0.041 \pm 0.020$, in agreement with the values for $t^{2}$ as estimated from the recombination lines.

Table 1 presents the $\mathrm{O}^{2+} / \mathrm{H}^{+}$ratio derived for each transition of multiplet $\mathrm{M}$ at each spatial position. Table 1 also presents the average $\mathrm{O}^{2+} / \mathrm{H}^{+}$ratio adopted for each position, the ratio of the $\mathrm{O}^{2+} / \mathrm{H}^{+}$abundance derived via the recombination lines to that derived via the collisional lines $\mathrm{O}^{2+}(\mathrm{recom}) / \mathrm{O}^{2+}$ (coll), the electron temperature derived via the [O III] 5007/4363 $\AA$ line ratio $\mathrm{t}_{\mathrm{e}}\left(\mathrm{O}\right.$ III) (in units of $10^{4} \mathrm{~K}$ ), and the implied $t^{2}$ based on the discrepancy between the recombination line and collisional line abundances.

Table 1

\begin{tabular}{llllll}
\hline$\lambda(\AA)$ & $\mathrm{M}$ & transition & CS posn & $\begin{array}{l}\mathrm{O}^{2+} / \mathrm{H}^{+} \times 10^{3} \\
6^{\prime \prime} \text { offset }\end{array}$ & $12^{\prime \prime}$ offset \\
\hline 4072.16 & 10 & $3 \mathrm{~d}^{4} \mathrm{~F}_{5 / 2}-3 \mathrm{p}^{4} \mathrm{D}_{3 / 2}$ & $1.15 \pm 0.08$ & $0.845 \pm 0.17$ & $1.22 \pm 0.24$ \\
4153.30 & 19 & $3 \mathrm{~d}^{4} \mathrm{P}_{5 / 2}-3 \mathrm{p}^{4} \mathrm{P}_{3 / 2}$ & $1.12 \pm 0.17$ & $0.784 \pm 0.12$ & \\
4119.22 & 20 & $3 \mathrm{~d}^{4} \mathrm{D}_{7 / 2}-3 \mathrm{p}^{4} \mathrm{P}_{5 / 2}$ & $1.34 \pm 0.13$ & $0.932 \pm 0.16$ & \\
4924.60 & 28 & $3 \mathrm{~d}^{4} \mathrm{P}_{5 / 2}-3 \mathrm{p}^{4} \mathrm{~S}_{3 / 2}$ & $1.35 \pm 0.20$ & $0.853 \pm 0.17$ & \\
4275.56 & 67 & $4 \mathrm{fF}[4]_{9 / 2}-3 \mathrm{~d}^{4} \mathrm{D}_{7 / 2}$ & $1.49 \pm 0.18$ & $1.11 \pm 0.22$ & \\
& & Adopted & $1.25 \pm 0.15$ & $0.905 \pm 0.18$ & $1.22 \pm 0.24$ \\
& \multirow{2}{*}{$\mathrm{O}^{2+}($ recom $) / \mathrm{O}^{2+}($ coll $)$} & 2.0 & 1.9 & 2.2 \\
& $\mathrm{t}_{\mathrm{e}}(\mathrm{OIII})$ & $0.900 \pm 0.010$ & $0.940 \pm 0.010$ & $0.940 \pm 0.010$ \\
& $t^{2}\left(\begin{array}{l}O I I \\
\text { abun }\end{array}\right)$ & $0.038_{-0.0090}^{+0.0068}$ & $0.040_{-0.0125}^{+0.0063}$ & $0.051_{-0.015}^{+0.014}$ \\
\hline
\end{tabular}

